\title{
Uso Da Plataforma Socrative Para Os Alunos De Química Orgânica: Um Estudo Preliminar
}

\author{
Maria Hosana Conceição', Olga M. Albuquerque², Ana Júlia Vilas Boas ${ }^{3}$ e Cristina M. Quintella ${ }^{4}$ \\ 1,2 e 3Universidade deBrasília (UnB), Brasil. '1hosanac@unb.br, ${ }^{2}$ olgamaria@unb.br, ${ }^{3}$ anajuliavilasboas@hotmail.com \\ ${ }^{4}$ Universidade Federal da Bahia (UFBA), Brasil, cris5000tina@gmail.com
}

\begin{abstract}
Resumo.Nos últimos anos, tem aumentado a busca por novas abordagens metodológicas aplicadas ao ensino de ciências devido, principalmente, ao uso de novas tecnologias que propiciam a autonomia e a motivação dos estudantes universitários. Assim, o objetivo principal dessa pesquisa foi avaliar o uso da plataforma, gratuita, Socrative para a disciplina de Química Orgânica. Trata de uma plataforma online na qual os estudantes praticam o conteúdo trabalhado em sala de aula usando os seus smartphones ou tablets. O estudo foi dividido em duas etapas, sendo que a primeira contou com a participação de sessenta estudantes de segundo semestre do curso de Farmácia, da disciplina de Química Orgânica, ofertada no $2^{\circ}$ semestre de 2019 . Cada uma das atividades consistiu em uma série de perguntas, de cada tópico, onde os estudantes deveriam responder através do Socrative. Os resultados das atividades indicaram que os estudantes foram receptivos ao uso da plataforma Socrative e reconheceram que a plataforma colaborou com o aprendizado dos conteúdos da disciplina. A segunda etapa, que trata da "pesquisa-ação", para obter o feedback da visão geral dos alunos em relação à disciplina ainda está sendo delineada. Até este momento, o estudo mostrou que a plataforma Socrative deve continuar sendo adotada na disciplina com adaptações do seu uso para melhor atender os estudantes.
\end{abstract}

Palavras-chave: Química Orgânica; Socrative; Inovação Docente.

\section{SOCRATIVE PLATAFORM USAGE BY ORGANIC CHEMISTRY STUDENTS: A PRELIMINARY STUDY}

\begin{abstract}
In recent years, the search for new methodological approaches applied to science teaching has increased, mainly due to the use of new technologies that provide the autonomy and motivation of university students. Thus, the main objective of this research was to use the free Socrative platform for the subject of Organic Chemistry. It is an online platform in which students practice the content worked in the classroom using their smartphones or tablets. The study was divided into two stages, the first with the participation of sixty students from the second semester of the Pharmacy course, of the subject of Organic Chemistry, offered in the 2nd semester of 2019. Each of the activities consisted of a series of questions, from each topic, where students should answer through the Socrative. The results of the activities indicated that the students were receptive to the use of the Socrative platform and recognized that the platform collaborated with the learning of the subject's contents. The second stage, which deals with "action research", to obtain feedback from the students' overview regarding the subject is still being outlined. So far, the study has shown that the Socrative platform should continue to be adopted in the discipline with adaptations of its use to better serve students.
\end{abstract}

Keywords: Organic Chemistry; Socrative; Teaching Innovation.

\section{INTRODUÇÃO}

Ao se tratar do ensino da química orgânica, os conceitos nem sempre são bem aceitos pelos estudantes e, além disso, os conteúdos de grupos funcionais e reações que, na sua maioria, podem ficar invisíveis aos olhos, os tornam difíceis e, até mesmo, promovem o 
desinteresse dos estudantes (Conceição, 2020) Segundo Soares (2018) (Soares, 2018) a aplicação de elementos lúdicos na educação, como por exemplo, jogos de química podem simplificar a abstração dos conteúdos e, conseqüentemente, atrair a atenção dos estudantes (Soares, 2018). Ainda em relação ao uso de jogos didáticos, no espaço da sala de aula, como ferramenta para a complementação e atualização dos recursos educativos, pesquisadoras da Secretaria de Estado da Educação e da Cultura do Estado do Rio Grande do Norte, Brasil propuseram um jogo intitulado "Dados Orgânicos" com o objetivo principal de auxiliar os estudantes, do Ensino Médio, no aprendizado das estruturas químicas e da nomenclatura IUPAC (International Union of Pure and Applied Chemistry) dos compostos orgânicos. Trata-se de um jogo de dados onde em cada face do cubo são colados nomes e estruturas químicas das moléculas orgânicas, permitindo aos jovens estudantes uma maior autonomia e motivação para o aprendizado da química orgânica (Souza \& Silva, 2012).

Domingos e Recena (2010) realizaram uma pesquisa, com abordagem qualitativa, para trabalhar os conteúdos de funções orgânicas oxigenadas e nitrogenadas. As professoras utilizaram como instrumentos de coleta de dados a observação e a gravação, em vídeos, das aulas em que houve o desenvolvimento das atividades e dos materiais produzidos pelos estudantes. As apresentações seguiram com perguntas e respostas de cada vídeo, permitindo concluir que houve a oportunidade, para os alunos, de reestruturação dos esquemas de assimilação e acomodação no processo de ensino e aprendizagem, possibilitando a construção dos conhecimentos em química orgânica (Araújo Domingos \& Piazza Recena, 2010).

\subsection{Justificativa}

Os cursos de graduação em Farmácia e Engenharia de Energia da Universidade de Brasília, Faculdade de Ceilândia (UnB/FCE) e Faculdade do Gama (UnB/FGA), respectivamente, têm a finalidade de formar profissionais aptos para se inserir no mercado de trabalho, tendo uma participação ativa no desenvolvimento da sociedade, particularmente nas decisões que envolvem o conhecimento químico. Além disso, tende a suprir este mercado cada vez mais exigente, no qual há um conjunto de metas a serem atingidas, que dizem respeito à formação técnica e humanística do profissional (Silveira, Flores, Pinho, \& Moura, 2010). Acredita-se que a tecnologia da informação possa ser utilizada como uma estratégia de ensino que permite desenvolver, com sucesso, um tópico ou conteúdo em sala de aula. Desta forma, a produção de novos softwares educacionais em todas as áreas do 
conhecimento tem sido, cada vez mais, objeto de pesquisas, provocando uma verdadeira revolução na concepção de ensino e aprendizagem (Barbosa et al., 2015).

O Laboratório de Inovações Tecnológicas para Ambientes de Experiência (ITAE) do Centro de Desenvolvimento Tecnológico da UnB - CDT-UnB, atua como um espaço de experiências, baseando-se em quatro pilares: educação, imersão, entretenimento e estética; assegurando que o aprendizado seja mais efetivo ao envolvermos os nossos sentidos num ambiente agradável e divertido. O Jogo ITAE é do tipo quiz e está disponível nos modelos de Quebra-cabeça ou Batalha naval (CDT, 2020).

Este cenário nos motivou a conhecer as pesquisas de desenvolvimento de ferramentas didático-computacionais na área de aplicativos multimídias realizadas pelos pesquisadores do Laboratório de Desenvolvimento de Softwares Educacionais (LDSE), da área de Ensino de Química, da Universidade Federal do Ceará (EQ/UFC) (Barbosa et al., 2015; da Silva Júnior et al., 2017). As ferramentas desenvolvidas pelos pesquisadores são simples e gratuitas e visam a melhoria do processo de ensino-aprendizagem de diferentes disciplinas do Curso de Química daquela Instituição, auxiliando professores e alunos, de todo o país e no mundouma vez que todos os aplicativos desenvolvidos estão disponibilizados na homepage do Laboratório de Desenvolvimento de Softwares Educacionais (http://www.ldse.ufc.br).

Nos últimos cinco anos, o LDSE se dedicou a reunir as características educacionais que utilizam softwares e jogos educacionais, resultando na criação do Stereogame (da Silva Júnior et al., 2017) . Nesse sentido, Frías e colaboradores (Frías, Arce, \& Flores-Morales, 2016) utilizaram a plataforma, gratuita, Socrative como uma ferramenta tecnológica de apoio à disciplina de Química Geral. Os resultados das atividades realizadas com os estudantes do $1^{\circ}$. ano de Odontologia demonstraram que a média de acertos foi de até $70 \%$, com uma boa receptividade dos estudantes para o uso da ferramenta tecnológica, medida através de questionário. Pintado e Cerio (2017) en la Universidad Publica de Navarra, Espanha, utilizaram a plataforma Socrative para apoiar o estudo autônomo do aluno no sentido de mantê-lo como o protagonista no processo da sua formação acadêmica (Pintado \& Cerio, 2017). Nesse sentido, o objetivo principal do estudo foi avaliar o uso da plataforma Socrative pelos estudantes da disciplina de Química Orgânica do curso de Farmácia da Universidade de Brasília, Brasília, DF, Brasil. 


\section{METODOLOGIA}

O estudo foi delineado em duas etapas distintas e interligadas. A primeira etapa, já concluida, se tratou do uso da plataforma Socrative, que é um aplicativo de perguntas e respostas baseado em nuvem. As duas características mais atraentes do Socrative são pela facilidade de uso, tanto para os professores quanto para os estudantes, bem como pelo fato de ser gratuito e pelo aplicativo poder baixado no smartphone, no tablet ou no laptop. Após a conclusão dos exercícios, é possível fazer o download dos resultados, em uma planilha do Excel ou enviar por e-mail (Frías et al., 2016; Manning, Keiper, \& Jenny, 2017). Para medir o grau de aceitação dos estudantes pela plataforma Socrative, foi aplicado um questionário de satisfação; contendo cinco perguntas e enviado pela plataforma Google forms. Quatro perguntas tiveram como finalidade tomar conhecimento da experiência do uso da tecnologia Socrative. A pergunta final foi orientada para uma autoavaliação a respeito do desempenho do estudante no curso.

A segunda etapa, ainda em construção, trata-se da pesquisa qualitativa do tipo "pesquisaação", cuja principal característica é realizar um projeto em que o próprio profissional (neste caso, a professora) procurará efetuar transformações em suas próprias práticas de ensino (Tripp, 2005). Para isto, a plataforma Socrative será também utilizada, mas com a pretenção de que os estudantes matriculados nos semestres 2020.1 e 2020.2 forneçam um feedback da eficiência da plataforma Socrative ao responder perguntas abertas considerando as fraquezas e pontos fortes da plataforma. As respostas dos estudantes serão categorizadas e tematizadas (Aslan \& Seker, 2017).

\section{RESULTADOS}

As atividades realizadas neste trabalho foram baseadas nos tópicos da disciplina de Química Orgânica. Foram aplicados, na plataforma Socrative, os exercícios de notação e nomenclatura dos hidrocarbonetos e das funções oxigenadas.

Ao serem consultados sobre a experiência prévia com a plataforma Socrative ou outra similar, 92\% responderam que não (Figura 1A), o que nos fez constatar que foi uma novidade para os estudantes. Nesse sentido, foram oferecidas cinco alternativas para que os estudantes definissem a sua experiência com a plataforma, numa escala do menor para o maior, as respostas se encontram na Figura 1B, com 40,5\% apontando como regular, $10,8 \%$ ótima e a mesma porcentagem para ruim; nos fazendo pensar que precisamos 
manter o uso da tecnologia para otimizar o seu uso. Quanto a satisfação em ter usado a plataforma em sala de aula, foi fornecida uma escala de 1 a 5 , onde 1 significava muito ruim e 5 muito boa, 15 estudantes (40,5\%), dos 37 respondentes, avaliaram como intermediária, com 2,7\% muito ruim e $8,1 \%$ muito boa (Fig. 1C). O resultando nos indicou que os estudantes receberam, satisfatoriamente, a utilização da plataforma Socrative. A Figura 1D nos indicou que os estudantes ainda têm dúvidas se o uso da plataforma os ajudou a compreender melhor os conteúdos da química orgânica, onde 40,5\% e 35,1\% reponderam regular e pouco, respectivamente e $13,5 \%$ reponderam não ter ajudado. Isso nos leva a crer que ainda é preciso investir tempo na preparação das questões para a plataforma e o seu alinhamento com as avaliações etc. Por outro lado, a aprovação na disciplina foi acima de $80 \%$ e nem todos os estudantes responderam o questionário de satisfação, somente 37 , dos 60 estudantes matriculados, responderam. Considerando que o questionário foi aplicado nos dias de 08 a 15 de abril de 2020 , durante o período do isolamento social pela pandemia da COVID-19, com as atividades da Universidade suspensas em função da necessidade do isolamento social, acreditamos que nem todos tiveram o acesso ao questionário.
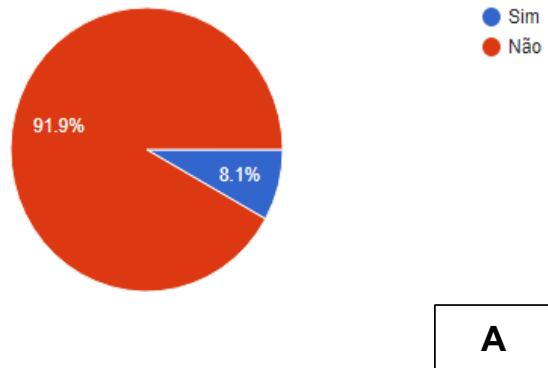

A

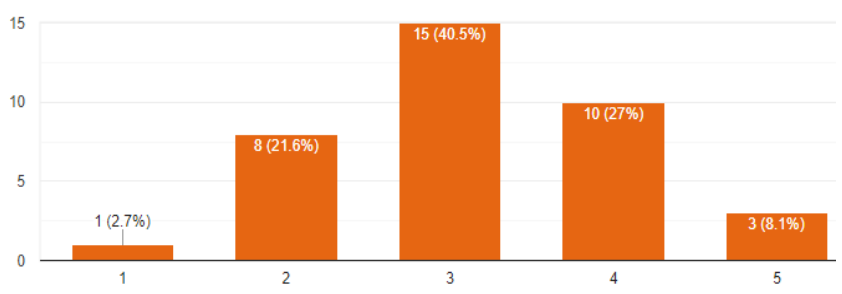

C

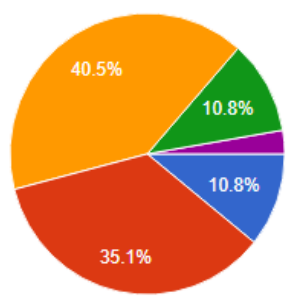

Ótima

Boa

Regular

Ruim

- Deve ser reformulado a forma de avaliação

\section{B}

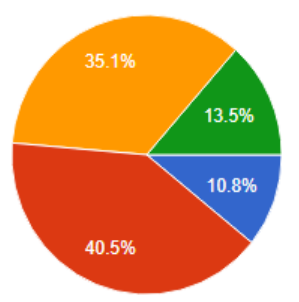

D

Figura 1. Resultados do Questionário de Satisfação dos estudantes com o uso da plataforma. A) experiência prévia com a sua utilização, B) experiência pessoal, C) valorização da aula e D) relação com os conteúdos da disciplina 


\section{CONCLUSÕES}

O foco principal deste trabalho foi o de incorporar uma nova tecnologia que permita manter a atenção e dar autonomia ao estudante para lograr um aprendizado mais próximo do contexto tecnológico social onde ele vive. Por isso, o artigo está sendo considerado como um estudo preliminar, de inovação docente, do uso da plataforma Socrative para estudantes do segundo semestre do curso de Farmácia da Faculdade de Ceilândia (FCE) da Universidade de Brasília (UnB) da disciplina de Química Orgânica. Como perspectiva, espera-se realizar o estudo qualitativo, da etapa 2, de entrevistas, onde iremos categorizar a percepção dos estudantes quanto ao uso da ferramenta tecnológica Socrative como um feedback para as ações da disciplina de Química Orgânica.

\section{REFERÊNCIAS}

Araújo Domingos, D.C., \& Piazza Recena, M.C. (2010). Elaboração de jogos didáticos no processo de ensino e aprendizagem de química: A construção do conhecimento. Ciências \& Cognição, 15, 272-281.

Aslan, B., \& Seker, H. (2017). Interactive response systems (irs) socrative application sample. Journal of Education and Learning, 6(1).

Barbosa, F.G., Mafezoli, J., Lima, M.A.S., Alexandre, F.S.O., Almeida, D.M.d., Leite Junior, A.J.M., \& Silva Júnior, J.N.d. (2015). Interactions: Design, implementation and evaluation of a computational tool for teaching intermolecular forces in higher education. Química Nova, 38, 1351-1356.

CDT, C.d.D.T.-.(2020). Laboratório de inovações tecnológicas para ambientes de experience - itae. http://www.cdt.unb.br/programaseprojetos/itae/index/?menu-principal=programas-e-projetos\&menuaction=itae.

Conceição, M.H. (2020). [Aulas de química orgânica para os cursos da saúde: Comunicação pessoal].

da Silva Júnior, J.N., Sousa Lima, M.A., Xerez Moreira, J.V., Oliveira Alexandre, F.S., de Almeida, D.M., de Oliveira, M.d.C.F., \& Melo Leite Junior, A.J. (2017). Stereogame: An interactive computer game that engages students in reviewing stereochemistry concepts. Journal of Chemical Education, 94(2), 248-250. doi: $10.1021 /$ acs.jchemed.6b00475.

Frías, M.V., Arce, C., \& Flores-Morales, P. (2016).Uso de la plataforma socrative.com para alumnos de química general. Educación química, 27, 59-66.

Haguenauer, C.J., Carvalho, F.S.d., Victorino, A.L.Q., Lopes, M.C.B.A., \& Filho, F.C. (2007). Uso de jogos na educação online: A experiência do latec/ufrj. Revista Educa online, 1(1), 1-14.

Manning, R.D., Keiper, M.C., \& Jenny, S.E. (2017). Pedagogical innovations for the millennial sport management student: Socrative and twitter. Sport Management Education Journal (Human Kinetics), 11(1), 45-54.

Pintado, A.B., \& Cerio, J.M.D.d. (2017). Socrative: Una herramienta para dinamizar el aula. Working Papers on Operations Management, 8(Splssue), 72-75.

FLSilveira, D., Flores, O., Pinho, D.L.M., \& Moura, M.A. (2010). Projeto pedagógico do curso de graduação em farmácia. In U. d. B.-.UnB (Ed.), (1a. ed., pp. 45). Universidade de Brasília http://fce.unb.br/comissoesfarmacia.

Soares, H.O. (2018). Desenvolvimento, aplicação e avaliação de um serious game como ferramenta auxiliar ao ensino de química (Mestrado), Instituto Politécnico de Setúal, Portugal. Retrieved from http://hdl.handle.net/10400.26/25229.

Souza, H.Y.S., \& Silva, C.K.O. (2012). Dados orgânicos: Um jogo didático no ensino de química. Holos, 3(28), 107-121.

Tripp, D. (2005). Pesquisa-ação: Uma introdução metodológica. Educação e Pesquisa, 31, 443-466. 